\title{
Flares in Dwarf Novae
}

\author{
Frank M. Bateson
}

Astronomical Research Ltd., P.O. Box 3093, Greerton, Tauranga, New Zealand

\begin{abstract}
This report provides a summary of flares observed visually in the dwarf novae V442 Cen, OY Car, TU Men and Z Cha. The flares precede an outburst by an average of around ten days.
\end{abstract}

\section{Introduction}

Members of the Variable Star Section, Royal Astronomical Society of New Zealand, have closely monitored most southern dwarf novae over the past five decades. The many hundreds of thousands of visual estimates include a number of definite observations of short lived flares which in the majority of cases preceded an outburst by a few days.

Bateson (1991a) suggested that these flares should be termed precursors to outbursts. He discussed in detail problems inherent in visual estimates and showed that he only accepted as flares those estimates that conformed to high standards of reliability.

Observations of V442 Cen, OY Car and TU Men were the first dwarf novae chosen to search the records for flares because these stars had minima well below the thresholds of the instruments used. A summary of the results are given below.

\section{Results}

V442 Cen: Type SS Cyg. Mean cycle 27.13 days (Bateson et al. 1991). Narrow and wide maxima differ in duration by little in mean brightness. Rise to outburst is steep and rapid. Minima are fainter than 16.0 mag.

A total of 19 flares were listed ranging in magnitude from 12.7 to 14.3 , all fainter than the mean outburst magnitude of 12.0 . These flares on average preceded an outburst by 8.8 days (range 4-14 days).

OY Car: SU UMa type. Superoutbursts have mean maximum magnitude of 11.35 ; mean width 11.5 days. Corresponding values for normal maxima are 12.38 and 1.8 days. Mean super cycle 328 days but this changes abruptly and varies within wide limits. Normal maxima follow superoutbursts at intervals of about 171 days (Bateson and Dodson 1983). Minimum magnitude is 17.5. 
Bateson (1991a) listed 11 possible flares of which two may have been normal outbursts. The magnitudes of the remainder ranged from 12.3 to 14.3 . These apparent flares preceded outbursts by intervals between 16 and 174 days excluding two long intervals probably caused by an outburst being unobserved. One flare preceded an outburst by 45 days and was then recorded at magnitude 12.3 fading to fainter than $\mathbf{1 3 . 5}$ within 5 minutes. This was probably an eclipse. TU Men: SU UMa type. The mean cycle length, maximum magnitude and outburst duration have all undergone abrupt and dramatic changes (Bateson 1991b). These followed two prolonged superoutbursts with durations of 20 and 24.6 days and maximum magnitudes of 11.8 and 11.6.

Seven possible flares were listed with maxima 13.2 to 14.2 . These occurred from 20 to 222 days before an outburst.

Z Cha: SU UMa type. Superoutbursts have a mean cycle of 217.6 days; mean maximum magnitude 12.1 ; mean duration 9.8 days. Corresponding values for normal outbursts are 51.3 days, $12.6 \mathrm{mag}$ and 0.92 days (Bateson 1991c). Minimum magnitude is $\mathbf{1 7 . 2}$.

Bateson (1991d) listed 26 possible flares of which two may have been of poorly observed normal maxima.

The magnitudes of the remainder ranged from 12.3 to 14.0. The intervals which these flares preceded an outburst were short, ranging from 2 to 73 days with one exception of 117 days.

\section{Conclusions}

There appears to be reliable evidence that flares occur in dwarf novae. The majority precede an outburst by an average of around 10 days.

\section{References}

Bateson F.M., 1991a, Publ. Var. Star Section, R. astr. Soc. N.Z. No. 16, 75

Bateson F.M., 1991b, Publ. Var. Star Section, R. astr. Soc. N.Z. No. 16, 40

Bateson F.M., 1991c, Publ. Var. Star Section, R. astr. Soc. N.Z. No. 17, 11

Bateson F.M., 1991d, Publ. Var. Star Section, R. astr. Soc. N.Z. No. 17, 74

Bateson F.M., Dodson, A.W., 1983, Publ. Var. Star Section, R. astr. Soc. N.Z. No. 11, 1

Bateson F.M., McIntosh, R., Brunt, D. 1991, Publ. Var. Star Section, R. astr. Soc.

N.Z. No. 16,4 